\title{
Transatlantica
}

Revue d'études américaines. American Studies Journal

1 | 2018

Slavery on Screen / American Women Writers Abroad: 1849-1976

\section{Carrie Hyde, Civic Longing: The Speculative Origins of U.S. Citizenship}

\section{Hélène Cottet}

\section{CpenEdition}

\section{Journals}

Édition électronique

URL : https://journals.openedition.org/transatlantica/11724

DOI : 10.4000/transatlantica. 11724

ISSN : 1765-2766

Éditeur

Association française d'Etudes Américaines (AFEA)

Référence électronique

Hélène Cottet, "Carrie Hyde, Civic Longing: The Speculative Origins of U.S. Citizenship », Transatlantica [En ligne], 1 | 2018, mis en ligne le 01 septembre 2019, consulté le 02 février 2023. URL : http:// journals.openedition.org/transatlantica/11724 ; DOI : https://doi.org/10.4000/transatlantica.11724

Ce document a été généré automatiquement le 2 février 2023.

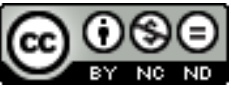

Creative Commons - Attribution - Pas d'Utilisation Commerciale - Pas de Modification 4.0 International - CC BY-NC-ND 4.0

https://creativecommons.org/licenses/by-nc-nd/4.0/ 


\title{
Carrie Hyde, Civic Longing: The Speculative Origins of U.S. Citizenship
}

\author{
Hélène Cottet
}

\section{RÉFÉRENCE}

Carrie Hyde, Civic Longing: The Speculative Origins of U.S. Citizenship, Cambridge, Harvard University Press, 2018, 308 p., \$46,50, ISBN 9780674976153

1 «I am a citizen of somewhere else » (Hawthorne, 1968, 44). C'est en butant contre cette déclaration célèbre de Hawthorne que Carrie Hyde a élaboré son enquête : impossible, en effet, de se rapporter à une définition claire de citizen qui permette d'élucider un contexte de référence impliqué par Hawthorne et partagé par ses lecteurs. En nous faisant part de cette anecdote (Hyde, 2018, 18-19), Hyde nous représente ses propres questionnements de lectrice, lesquels ont toute leur importance dans cet ouvrage qui revendique une approche textuelle de l'histoire de la citoyenneté («to return citizenship to the acts of reading and writing ", 7). Par ailleurs, elle restitue le caractère proprement déroutant du constat dont elle s'empare dans son livre: l'absence de définition juridique du statut de citoyen des Etats-Unis avant le Civil Rights Act de 1866, dont les dispositions intègrent la Constitution à l'intérieur du XIVe amendement en 1868. En 1868 toujours, l'Expatriation Act apporte d'autres éléments cruciaux sur la possibilité de se défaire de cette citoyenneté.

2 Carrie Hyde insiste donc sur ce vide juridique qui dure près d'un siècle après l'indépendance des Etats-Unis, "when the cultural and juridical meaning of citizenship - as well as its scope - was under-articulated and open to debate » (1). Elle va étudier cette période précisément pour démontrer qu'en l'absence de définition juridique le terme de «citoyen » n'en est pas moins largement mis en circulation, et que la valeur même de cette catégorie politique est élaborée par les discours et débats qui s'en emparent, quand bien même ils ne décident encore de rien. Il faut alors admettre le mode "spéculatif» ou encore «subjonctif» des définitions de la 
citoyenneté qui ont cours avant 1868: l'analogie proposée par Hyde avec le mode grammatical «subjonctif » (16-17) dit bien en effet un discours qui a conscience de sa propre irréalité, d'un décalage avec les faits, et qui exprime un souhait. Pour Carrie Hyde, ces discours subjonctifs revêtent souvent un caractère stratégique, car ils sont des moyens d'infléchir une définition encore en cours : «Their political efficacy resides in subjunctive formulations - where the possible (what might or could be) and the prescriptive (what should or ought to be) collide in language that seeks to compel by persuasion » (16). L'expression « civic longing " (ou encore «the longing to belong ", 9) présente dans le titre fonctionne de manière similaire: il ne s'agit pas en effet de considérer "qui a la citoyenneté » et « qui ne l'a pas », pour Hyde, c'est le désir même d'appartenance, ou, dans certains cas, de désengagement, qui aurait façonné la notion de citoyenneté - et ce de manière à la fois préliminaire et durable.

La période qu'elle étudie ne serait donc pas vide mais pleine, si l'on considère que le caractère encore sous-déterminé de la citoyenneté américaine permet une réélaboration continue de son sens. Un tel règne des possibles ne signifie pas, par ailleurs, que le statut de citoyen ait été uniquement imaginé ou interprété dans une visée émancipatrice, car l'absence de cadre juridique clair a permis aussi bien l'articulation de conceptions très étriquées d'une appartenance politique. Toutes les propositions examinées par Hyde informent néanmoins la catégorie "citoyen », et cela, largement en-dehors de textes juridiques, c'est-à-dire de discours ayant une véritable efficacité garantie par l'état. C'est l'adjectif «extralegal» (traduit ici par «extrajuridique ») qui permet à l'auteur de définir deux particularités de ses sources :

I use the term «extralegal » in two ways : both, in a simple sense, to collectively describe a number of nonlegal traditions of political authorization, and in a more specialized sense, to highlight the negative definitional impulse that has sustained and fueled the almost melancholic idealization of citizenship from its changeable outer limits. (9)

4 De fait, c'est le passage par la négative - par des figures de non-citoyens, ou par des «ailleurs " jugés soit plus hospitaliers que le territoire états-unien, soit, à l'inverse, inhospitaliers - qui contribue à fantasmer ou à idéaliser le statut du citoyen, à l'intérieur d'un imaginaire puissant qui reste culturellement actif.

«In this spirit, I read the imaginative traditions of citizenship as historically activated genres of political theory " (9) : c'est à l'occasion d'une introduction très denseparfois trop, à l'image de cette dernière citation, lorsqu'à force de densité la langue de Hyde perd en clarté - que l'auteur précise son corpus et la méthode de son interprétation. En évoquant « les traditions imaginatives de la citoyenneté », Hyde veut prendre au sérieux la puissance de théorisation, mais aussi l'influence historique, de discours qui s'éloignent ostensiblement du réel. Elle ouvre alors un questionnement méthodologique plus vaste: "the early history of citizenship asks us to think expansively about the kind of documents that are integral to politics» (11). C'est en particulier une réhabilitation de la portée politique de la littérature que Hyde défend, à partir d'un processus d'historicisation: il s'agit en effet de percevoir quels sont les discours reçus de manière politique à l'époque qu'elle étudie, et notamment de prendre en compte combien la littérature états-unienne, tant à l'école qu'en dehors, recevait une importance politique. Elle ajoute : «The decentralized, extralegal development of citizenship offers an instructive opportunity for reexamining what it means to think of the imaginative arts as properly political - rather than normatively social or descriptively historical. » S'appuyant sur The Public and Its Problems de John Dewey, 
Hyde propose en effet de se départir des modalités indexiques selon lesquelles art et histoire sont articulés : l'art n'est pas seulement le véhicule d'une information, situable en son dehors, nous renseignant par exemple sur une réalité historique, il est le lieu où se jouent des manières de voir en commun et, à ce titre, il a pu être un outil de persuasion politique (12). En ayant l'occasion d'aller chercher ailleurs que dans un débat juridique les discours qui infléchissent une première définition de la citoyenneté américaine, Hyde a donc pleinement conscience de réviser les règles courantes des "échanges " entre littérature et histoire, et propose de nouvelles réflexions sur la portée politique de la littérature. Cette dimension théorique de l'ouvrage n'est pas le moindre de ses apports, d'autant que Hyde synthétise en passant bien des débats, et nous fait bénéficier de l'impressionnante somme de ses lectures.

6 Carrie Hyde commence son étude par un rappel du cadre législatif qui, en 1868, mettra un terme aux spéculations en définissant enfin clairement le statut de citoyen des Etats-Unis. Il faut en effet bien prendre en compte ce qui se décide au sein du XIVe amendement à la Constitution et de l'Expatriation Act pour prendre la mesure des différents sujets qui restaient, auparavant, ouverts. On peut souligner en particulier deux points importants qui sont verouillés avec l'encadrement juridique d'une citoyenneté nationale : le droit du sol l'emporte sur le droit du sang, si bien qu'en dépit de servitudes passées les Africains-Américains sont reconnus comme citoyens; l'Expatriation Act formalise un modèle d'allégeance volontaire de la citoyenneté étatsunienne, où l'on en vient à une réciprocité du contrat citoyen-Etat qui n'existait pas au préalable.

7 Si cette réciprocité n'était pas juridiquement établie alors, Hyde montre pourtant qu'elle existait, à l'état de croyance, pourrait-on dire, chez un écrivain comme David Ramsay, l'un des premiers historiens de la Révolution américaine, et auteur de l'ouvrage Dissertation on the Manner of Acquiring the Character and Privileges of a Citizen in the United States en 1789. Ce texte est en effet influencé par la philosophie politique de John Locke et le concept du contrat - pourtant la notion de « citoyen » elle même n'est pas utilisée par Locke, mais dénote l'influence de Rousseau. De manière générale, c'est bien Rousseau par le biais de la Révolution française et de la Déclaration des droits de l'homme et du citoyen qui influence un remplacement outre-atlantique de «sujet » par "citoyen". Là où le premier est progressivement redéfini par la relation d'assujettissement à laquelle il se soumettrait, la citoyenneté est idéalisée comme l'appartenance politique qui confère des « droits » à qui la détient.

8 Ramsay, pour sa part, adoptait donc une lecture Lockienne qui, pour l'heure, ne coïncidait pas avec une loi fédérale. De manière plus générale, à l'intérieur de cette période formatrice, quiconque essaie de clarifier le statut de citoyen américain met essentiellement en avant ses propres choix de lecture. Carrie Hyde revient notamment sur les propositions faites par le procureur général Edward Bates en 1862, lorsqu'il tente de répondre à la question : est-ce que les hommes noirs peuvent être citoyens américains ? Remarquant qu'il n'existe pas de sources faisant autorité, Bates offre sa propre interprétation textualiste de la loi: en l'occurrence, l'absence de définition claire est reçue par lui comme une opportunité, autorisant tout à fait d'imaginer une citoyenneté des Noirs américains. Cette lecture va à l'encontre de celle fournie précédemment par Roger Taney, Chief Justice à la Cour suprême des Etats-Unis, dans la décision rendue en 1857 pour Dred Scott v. Sandford, qui excluait « la race africaine asservie » de la citoyenneté1. C'est ici selon la théorie de l'« intention originelle » qu'il 
faut comprendre la lecture faite par Taney du document sur lequel il décide de fonder sa décision, la Déclaration d'indépendance - Taney prétendant, au regard de divers éléments contextuels et extrapolations, qu'il était impensable pour les pères fondateurs de prévoir que les Noirs américains soient concernés par leur document.

9 La décision dite Dred Scott fut très importante, mais Hyde explique qu'elle ne peut pour autant être tenue pour représentative des efforts fournis lors de cette période pour définir le statut de citoyen, notamment si on la place en regard des premières constitutions de certains états qui, elles, étaient inclusives. Carrie Hyde se saisit donc de ces différents exemples pour mettre en garde de manière générale contre toute lecture "originaliste", biais que l'on peut conserver même en tant qu'historien ou historienne lorsque les enjeux actuellement charriés par tel ou tel effort de définition sont projetés dans la source elle-même. Hyde sera notamment passée plus tôt par l'exemple des Amérindiens pour bien indiquer que, dans leur cas, l'émancipation passait par des revendications de "souveraineté » qui prennaient largement le pas sur un fantasme de citoyenneté. Il existait, autrement dit, plusieurs manières d'envisager l'appartenance politique, autant de nuances que l'on tend à effacer si l'on interpose entre la source et son interprétation la valeur que nous accordons aujourd'hui à la notion de citoyenneté.

Si un retour aux pratiques d'interprétation de Ramsay, Bates, ou Taney, permet donc de souligner le caractère spéculatif de leurs lectures, la deuxième partie de l'ouvrage montre comment, en l'absence de texte de référence clair, il est un livre, la Bible, qui a $\mathrm{pu}$ faire autorité en proposant une autre version de la citoyenneté. La loi divine se substitue, alors, à la loi des hommes, et la citoyenneté chrétienne implique, semble-t-il, un renoncement à la politique. C'est là le phénomène qu'étudie Carrie Hyde en s'intéressant à la circulation d'une traduction - concurrente à celle de la King James Version - d'un épître de Saint Paul aux Philippiens où la notion de citoyenneté apparaît : « nous avons notre citoyenneté dans les cieux, d'où nous attendons comme sauveur le Seigneur Jésus Christ » ( "we are citizens of heaven, from which we earnestly expect a savior, the Lord Jesus Christ ", Thomson, 1808, Phil. $3: 19-21)$. Comme elle l'explique, l'allégeance aux cieux dépend d'une rupture d'allégeance envers la nation, mais c'est là un déplacement à double tranchant, particulièrement dans le cas des Noirs américains : il peut être signe de fatalisme - renoncement à atteindre ici et maintenant des droits qui seront octroyés dans une autre vie - aussi bien que de résistance - il autorise la critique des institutions humaines au vu d'un modèle religieux. D'autre part, Hyde s'intéresse au développement d'un nationalisme chrétien aux Etats-Unis qui complique encore ce recours à la Bible, puisque, de fait, il s'agit alors d'envisager un royaume de Dieu sur terre, et l'avènement d'une citoyenneté chrétienne ici-bas. Comme elle le note, "political commentators who successfully fused these two strains of Christian thought were able to repurpose the otherwise merely fatalistic metaphor of heavenly citizenship as a theological call to arms and reform. » (Hyde, 2018, 47) Dans ce deuxième grand temps de l'ouvrage, Hyde étudie donc des textes dans lesquels le christianisme devient l'instrument d'une critique de l'esclavage. Elle s'attarde notamment sur Appeal to the Coloured Citizens of the World, de l'abolitionniste noir David Walker (1829), et, ce qui est assez attendu, sur l'œuvre de Harriet Beecher Stowe, choisissant cependant non pas Uncle Tom's Cabin mais Dred, son second roman abolitionniste, datant de 1856. C'est en particulier dans le cas de ce second texte qu'elle démontre les usages critiques ambigüs d'une notion de citoyenneté empruntée à la Bible, qui permet aussi bien de retarder toute justice que de juger les défaillances des 
Etats-Unis, et, ici, permet surtout à l'auteur de constituer tous Chrétiens, noirs ou blancs, en co-citoyens.

11 C'est encore aux exclus politiques, qui gagneraient à une définition de la citoyenneté dégagée des lois temporelles états-uniennes, que Hyde s'intéresse dans la suite de cette partie, prêtant attention cette fois à l'usage rhétorique qui est fait non plus de la « loi divine » mais d'autres « lois supérieures ", les « lois de la nature ». L'objet dont elle part est ici la révolte d'esclaves ayant eu lieu à bord du Créole, en 1841 : 135 esclaves sont libérés lorsqu'ils accostent aux Bahamas, territoire britannique où l'esclavage avait été aboli en 1834. Un tel événement donne soudainement à voir la traversée d'une frontière au-delà de laquelle l'esclavage se révèle dans toute son aberration. L'océan par lequel les esclaves ont accédé à leur liberté devient ainsi représentatif d'une nature qui n'admet pas l'esclavage, et fragilise l'échelle locale à laquelle il persiste. Hyde met ici en regard des textes de Daniel Webster, Secrétaire d'État au moment des faits, et de Frederick Douglass - passant notamment par sa seule œuvre de fiction, publiée en 1852, The Heroic Slave, a heartwarming Narrative of the Adventures of Madison Washington, in Pursuit of Liberty. Il s'agit de comparer les directions inverses dans lesquelles ils mènent l'évocation d'une loi de la nature pour dé-légitimer ou légitimer la révolte des esclaves. Les forces du climat ou l'océan lui-même donnent lieu aux métaphores qui soustendent leurs textes, et chez Douglass permettent de souligner le caractère circonscris et local de l'esclavagisme. La portée critique d'une loi naturelle dans le discours est donc qu'elle vient autoriser la révolte et met en scène, au mode subjonctif, l'existence politique de personnes privées de droits.

12 Il existe une modalité moins prescriptive du subjonctif, ayant trait au domaine du possible, que Hyde explore dans sa troisième et dernière partie à travers la notion de « fictivité » (« fictionality - the epistemic mode and problem of the fictive », 117). Il est ici plus pleinement question de littérature, et des relations spécifiques entre textes littéraires et politique. Il semble d'abord qu'un rapprochement puisse être fait entre le désintéressement, ou désengagement, chrétien et l'autonomie qui peut être accordée à la littérature. Pourtant, le croyant tient l'alternative d'une citoyenneté aux cieux comme véritable ou réelle, là où le lecteur se projetant dans l'univers autre de l'œuvre de fiction n'a pas cette foi en l'existence du monde dépeint. L'une des thèses fortes de Hyde sera alors de dire que c'est parce que la fiction échoue, d'une certaine façon, à concevoir une « alternative habitable » (« an inhabitable alternative »,121) aux réalités politiques du présent qu'elle pourra rediriger le lecteur vers la politique et une reconsidération de celle-ci.

13 Hyde veut ici s'intéresser plus directement à un art qui proclamerait sa coupure d'avec le monde, son absence de valeur référentielle, et elle contourne alors deux possibilités : celle d'une valeur politique de la fiction réaliste, la plus raisonnable au premier abord, ou celle d'une radicalité politique prêtée d'emblée à tout art "autonome ", qui critiquerait forcément l'histoire dont il se détache : "Critics sometimes discuss the overblown unreality of the romantic aesthetic as a kind of "aesthetic dissent," wherein the imagination's departure from history is itself a form of radical protest or revolt » (122). Elle se penche ici tout particulièrement sur Nathaniel Hawthorne, qui dans «The Custom House ", texte utilisé comme introduction à The Scarlet Letter, et l'un des lieux principaux où l'auteur développe sa théorie du genre littéraire de la "romance ", déclarait : «I am a citizen of somewhere else ». La formule proclame une indépendance qui ressemble au renoncement chrétien à ce monde, mais la notion de citoyenneté n'en 
projette pas moins cet ailleurs selon le modèle de l'appartenance politique. Comme le montre Hyde, c'est toute la subtilité de la « romance » telle que conçue par Hawthorne que d'être cette écriture liminale entre l'ailleurs et l'ici, et qui nous retourne au monde par le biais même de la défamiliarisation. Il n'est donc pas question de nier le monde, ni d'être absent de la politique, mais de la suspendre, et c'est ainsi que Hyde interprète également la notion de neutralité chère à Hawthorne. Être citoyen d'un ailleurs, ce n'est pas non plus s'affranchir des réalités politiques, mais, ainsi que l'interprète Hyde, précisément remettre en question la doctrine britannique de l'allégeance perpétuelle à la nation qui avait cours avant le passage de l'Expatriation Act, et s'extraire du « sol » qui conditionnerait d'emblée l'appartenance. C'est donc, en somme, prendre poétiquement une position politique.

Hyde montre d'autre part que Hawthorne, en accentuant dans certains de ses contes, "The New Adam and Eve" par exemple, la virtualité même du monde fictif qu'il propose, nous préparerait d'autant mieux à en revenir, comme d'un exil : à en revenir mus par la nostalgie, précisément, du monde que nous aurions laissé derrière nous en lisant. Il y aurait ainsi une ré-affiliation politique permise par la fiction, sur le mode du désir ("longing»). Mais l'artifice même auquel le texte aura exposé son lecteur aura défait, du même coup, tout ce qui pouvait sembler naturel dans cette appartenance, c'est-à-dire dans le lien entre citoyen et état.

Ayant esquissé avec Hawthorne certains des chemins les plus paradoxaux sur lesquels littérature et politique sont amenés à s'entre-croiser, Hyde passe ensuite à un tout autre cas en explorant les usages didactiques de la littérature américaine. Sa dernière étude porte ainsi sur un texte moins canonique en apparence que ceux de Hawthorne mais qui, quoiqu'écrit à l'occasion de la guerre civile, a eu une longévité exceptionnelle, notamment dans les salles de classe. Il s'agit de The Man Without a Country (1862), une nouvelle d'Edward Everett Hale qui porte sur la traîtrise et sur la punition imaginaire du coupable, condamné à errer toute sa vie sur les océans sans jamais plus entendre le nom même de sa patrie. Il est un exemple parfait de negative instruction, c'est-à-dire du potentiel éducatif offert par le contre-modèle. A ce titre, le texte offre aussi un exemple parfait de ce que Hyde entend par l'idéalisation de la citoyenneté : on y voit des personnes qui en sont privées - traîtres, exilés, esclaves - et c'est à partir d'eux que le caractère désirable de la citoyenneté apparaît, sans qu'il soit besoin d'aucune précision sur la nature exacte des droits et privilèges du citoyen. À nouveau, c'est sur un mode résolument nostalgique que se joue le désir d'appartenance. Ici, la sous-détermination $\mathrm{du}$ statut du citoyen est décisive pour en élaborer la valeur même. Mais comme le montre Hyde, les limites inévitables d'une instruction par la négative tiennent à sa réception, qui peut déjouer les intentions de départ. Ici, c'est le caractère tyrannique et injuste de la punition qui a pu, au contraire, rendre peu souhaitable l'appartenance à l'état capable de l'infliger, et c'est alors la sympathie ressentie pour l'exilé qui aura pu s'étendre à d'autres personnes privées de droit, notamment les esclaves transitant eux aussi par les mers et à qui le traitre est implicitement comparé.

16 Le mode subjonctif propre aux discours étudiés par Hyde aura donc mis au jour un travail politique effectué par une « déréalisation » du statu quo - qui entretient le débat tout en paraissant s'y dérober. Revenant sur le corpus qu'elle constitue dans l'ouvrage, on peut remarquer, en sus, que les textes extra-juridiques dont part Hyde ont tous en commun d'évoquer une extra-territorialité où se déploient vraiment les différentes possibilités d'une citoyenneté. Qu'il s'agisse du royaume des cieux, des océans 
internationaux sur lesquels vogue le Créole, de l' "ailleurs» de Hawthorne, ou de l'homme sans patrie voguant lui aussi sur les mers, il s'agit bien à chaque fois de se déporter en dehors du sol états-unien pour interroger la politique américaine. Ainsi que le note Hyde, il semble tout à fait paradoxal qu'aussi bien le christianisme ou la romance hawthornienne soient très rapidement devenus les marqueurs d'une certaine américanéité. On peut s'interroger sur cette faculté à naturaliser ou "ré-affilier ", en quelque sorte, des pensées qui paraissaient se déprendre nettement d'un enracinement national, et il apparaît surprenant que Hyde ne se soit pas plus systématiquement interressée à ce phénomène: si elle considère qu'un nationalisme chrétien, par exemple, transforme considérablement le sens donné aux invocations religieuses de la " citoyenneté ", qu'advient-il des significations politiques qu'elle donne à la romance dès lors que Hawthorne est lui aussi lu comme un écrivain national ? C'est en fait le maniement de la notion d' "autonomie ", rapportée à la littérature américaine, qui reste un point malaisé dans l'ouvrage, tant il demeure attendu, au cours du XIX siècle, que la littérature ait une pertinence pour la jeune nation, soit prise dans un rapport transitif à celle-ci, ce que montrent par exemple les usages scolaires et civiques du texte de Hale. Hawthorne, quoiqu'il soit à l'origine de l'enquête menée, reste néanmoins un cas limite à l'intérieur de celle-ci, à partir de la singulière position dedans-dehors qui fut la sienne et qui n'est pas toujours raccordée aux partis-pris méthodologiques du reste de l'ouvrage.

La cohérence de Civic Longing n'en reste pas moins dans son panorama des « ailleurs de la citoyenneté » (" the elsewhere of citizenship », pour reprendre le titre éloquent d'un des chapitres), une somme spéculative qui nous restituerait presque une alternative juridique. C'est peut-être en effet en repartant de la distinction appuyée par Hannah Arendt entre lex romaine et nomos grec à partir de leurs étymologies - lex, la loi comprise comme rapport entre les hommes et accord entre eux ; nomos, la loi d'ores et déjà rapportée à une action de répartir, résider, établir les frontières (Arendt, 1961, 104) - que l'on est tenté de redéfinir la plupart des sources extra-juridiques exploitées par Hyde comme des sources alter-juridiques. Ce qui s'y fantasmerait, ne pourrait-ce alors pas être une conception possible de la loi comme lex - fondement du rapport même entre les hommes-, si bien que la sous-détermination de la notion de citoyenneté aurait permis, pendant un temps, de la soustraire aux seules logiques de territoire? Si l'on se rappelle que le terme même de "citoyen » est emprunté à un épisode de l'histoire française - mais que la Déclaration des droits de l'homme et du citoyen composait elle-même avec l'exemple de la Déclaration d'indépendance américaine - on perçoit d'autant mieux combien l'émergence du terme échappe aux nationalités, ce qui peut aujourd'hui sembler paradoxal. En mettant audacieusement au centre de son étude des pratiques d'écriture et, surtout, de lecture, Hyde accentue de fait cette instablité de la citoyenneté, et nous restitue ainsi toute l'amplitude d'un désir d'appartenance. 


\section{BIBLIOGRAPHIE}

ARENDT, Hannah, La Condition de l'homme moderne, Paris, Calmann-Lévy, 1961.

Dred Scott v. Sanford : A Brief History with Documents, dir. Paul Finkelman, New York, Bedford, 1997.

HAWTHORNE, Nathaniel, The Scarlet Letter, Centenary Edition of the Works of Nathaniel Hawthorne, Columbus, Ohio State University Press, 1968.

THOMSON, Charles, trad., The Holy Bible, Containing the Old and New Covenant, commonly Called the Old and New Testament : Translated from the Greek, Philadelphie, Jane Aitken, 1808.

\section{NOTES}

1. «But it is too clear for dispute, that the enslaved African race were not intended to be included, and formed no part of the people who framed and adopted this declaration; for if the language, as understood in that day, would embrace them, the conduct of the distinguished men who framed the Declaration of Independence would have been utterly and flagrantly inconsistent with the principles they asserted; and instead of the sympathy of mankind, to which they so confidently appeared, they would have deserved and received universal rebuke and reprobation. » (Dred Scott v. Sanford, 1997, p. 63)

INDEX

Thèmes : Recensions

\section{AUTEURS}

\section{HÉLÈNE COTTET}

MCF, Université de Lille 